\title{
Hidden Quantum Group Symmetry and Integrable Perturbations of Conformal Field Theories ${ }^{\star}$
}

\author{
N. Reshetikhin ${ }^{1}$ and F. Smirnov ${ }^{2}$ \\ ${ }^{1}$ Mathematics Department, Harvard University, Cambridge MA 02138, USA \\ ${ }^{2}$ LOMI, Fontanka 27, Leningrad, 191011, USSR
}

\begin{abstract}
The hidden quantum group symmetry in the quantum Sine-Gordon model is found. This symmetry provides the possibility to restrict the operator algebra of the model to subalgebras. It is shown that these subalgebras are massive deformations of minimal conformal field theories.
\end{abstract}

\section{Contents}

Introduction . . . . . . . . . . . . . . . . . . . . . 157

0. Quantum Group $S L(2)_{q}$. . . . . . . . . . . . . . . . . 158

1. $S L(2)_{q}$ Invariance of Sine-Gordon Models. . . . . . . . . . . 162

2. Reductions of Sine Gordon for Rational $\xi / \pi$. . . . . . . . . . 168

3. Ultraviolet Limit of RSG . . . . . . . . . . . . . . . . 173 References. . . . . . . . . . . . . . . . . . . 177

\section{Introduction}

The goal of the present paper is to describe an interesting property of certain integrable models: Invariance with respect to the action of quantum groups. This invariance is essentially the quantum effect and provides the possibility to restrict the operator algebra of the models to subalgebras. Most importantly ultraviolet asymptotics of Green functions in the restricted subalgebras are described by conformal field theories [1] different from the one associated to the model itself.

In this paper we concentrate on the case of quantum Sine-Gordon (SG) model [2]. The model is described by the Lagrangian

$$
\mathscr{L}=\int\left(\frac{1}{2}\left(\partial_{\mu} \varphi\right)^{2}+m^{2} \cos \sqrt{\gamma} \varphi\right) d x .
$$

We use the renormalized coupling constant $\xi=(\pi \gamma) /(8 \pi-\gamma)$. The spectrum of the models contains solitons and (for $\xi<\pi$ ) their bound states - breathers $[3,4]$. From the viewpoint of papers [5-7], SG is the perturbation of the conformal field theory

\footnotetext{
* Supported in part by the Department of Energy under Grant DE-FG02-88ER25065
} 
(CFT) with $c=1$ (massless free bosons) by the operator with scaling dimensions $(\Delta, \bar{\Delta})=(\gamma / 8 \pi, \gamma / 8 \pi)$. The other integrable quantum models are discussed in the conclusion.

We show that the $S$-matrix of the model commutes with the actions $T_{R}$ and $T_{L}$ of quantum groups $S L(2)_{q}$ and $S L(2)_{\bar{q}}\left(q=\exp \left(2 \pi^{2} i / \xi\right)\right)$. This on-shell symmetry can be spread to off-shell objects. Namely, we show that there are two subalgebras of the local operator algebra of SG $\left(A_{R}^{\mathrm{inv}}, A_{L}^{\mathrm{inv}}\right)$ which are invariant with respect to $T_{R}, T_{L}$. It is known [8] that the SG model is equivalent to the massless two-component Thirring model. The coupling constant of SG describes the anisotropy in the Thirring model. The case $\gamma=8 \pi$ corresponds to isotrophical interaction. Our quantum group symmetry describes the degeneracy of the spectrum in these models, and it becomes the ordinary $S U(2)$-symmetry at $\gamma=8 \pi$.

Then we consider rational values of $\xi / \pi$. Due to special proerties of $S L(2)_{q}$ for $q^{r}=1$, it is possible to reduce the space of states preserving the locality of invariant operators. Following [9], the reduced model is denoted by $\operatorname{RSG}(\xi / \pi)$. We show using this fact that SG Green functions of invariant operators coincide with RSG Green functions. We study the physical interpretation of $\operatorname{RSG}(\xi / \pi)$. The $S$-matrices in these models are of RSOS type [10-12]. These $S$-matrices are unitary only for the following values of coupling constants:

$$
\frac{\xi}{\pi}=\frac{p \pi}{p k+1}, p=2, \ldots, k=0, \ldots, \frac{\xi}{\pi}=\frac{3 \pi}{3 k+2}, \quad k=0, \ldots
$$

Only for $\xi=\pi p$ in addition to the unitarity of $S$-matrices, the algebra of local operators appears to be closed with respect to the operation of Hermitian conjugation. In other cases there are two conjugated algebras of local operators which are not mutually local. We call this situation *-violated, it was discussed in [9] for $\xi=2 \pi /(2 n+1)$.

Finally we present arguments in favor of the conclusion that the model $\operatorname{RSG}\left(p_{1} /\left(p_{2}-p_{1}\right)\right)$ coincide with the perturbations of $M_{p_{1} / p_{2}}$ CFT [1] by the operator $\phi_{1,3}$ [5]. As it has been mentioned, the Green functions in RSG coincide with those of invariant operators in SG. This fact implies that the short distance behavior of certain SG Green functions is described by $M_{p_{1} / p_{2}}$. In usual $(c=1)$ scaling limits these Green functions disappear because the scaling dimensions in $M_{p_{1} / p_{2}}$ are less than the corresponding scaling dimensions for $c=1$. The case $\xi=\pi \rho$ corresponds to the minimal unitary [13] CFT $M_{p / p+1}$.

\section{Quantum Group $S L(2)_{q}$}

Here we remember the definition of quantum group $S L(2)_{q}$ and give some important facts about this algebra. This is the deformation of the algebra of functions over $S L(2)$ [14-16]. It can be defined as an associative algebra with generators $g_{\varepsilon^{\prime}}^{\varepsilon}, \varepsilon, \varepsilon^{\prime}= \pm 1$. Let $g=\left(\left(g_{\varepsilon^{\prime}}^{\varepsilon}\right)\right)$ be a matrix of generators and $q$ some formal parameter. Relations in the algebra $S L(2)_{q}$ can be written in the so-called $R$-matrix form:

$$
\begin{aligned}
& R(q) g_{1} g_{2}=g_{2} g_{1} R(q), \\
& g_{1}^{1} g_{2}^{2}-q^{1 / 2} g_{1}^{2} g_{2}^{1}=1
\end{aligned}
$$


Here $g_{1}, g_{2}, R(q)$ are matrices acting in $\mathbb{C}^{2} \otimes \mathbb{C}^{2}, g_{1}=g \otimes 1, g_{2}=I \otimes g, g$ is the matrix of generators and

$$
R(q)=\left(\begin{array}{cccc}
q^{1 / 2} & 0 & 0 & 0 \\
0 & 1 & q^{1 / 2}-q^{-1 / 2} & 0 \\
0 & 0 & 1 & 0 \\
0 & 0 & 0 & q^{1 / 2}
\end{array}\right)
$$

Algebra $S L(2)_{q}$ is the Hopf algebra [14] with comultiplication $\Delta: S L(2)_{q} \rightarrow S L(2)_{q} \otimes$ $S L(2)_{q}$

$$
\Delta\left(g_{\varepsilon^{\prime}}^{\varepsilon}\right)=\sum_{\varepsilon^{\prime \prime}} g_{\varepsilon^{\prime \prime}}^{\varepsilon} \otimes g_{\varepsilon^{\prime}}^{\varepsilon^{\prime \prime}} .
$$

The antipode $S: S L(2)_{q} \rightarrow S L(2)_{q}$ is defined as

$$
S(g)=\left(\begin{array}{cc}
g_{2}^{2} & -q^{-1 / 2} g_{2}^{1} \\
-q^{1 / 2} g_{1}^{2} & g_{1}^{1}
\end{array}\right) .
$$

It plays the role of inverse matrix to $g$ :

$$
\sum_{\varepsilon^{\prime \prime}} g_{\varepsilon^{\prime \prime}}^{\varepsilon} S\left(g_{\varepsilon^{\prime}}^{\varepsilon^{\prime \prime}}\right)=\sum_{\varepsilon^{\prime \prime}} S\left(g_{\varepsilon^{\prime \prime}}^{\varepsilon}\right) g_{\varepsilon^{\prime}}^{\varepsilon^{\prime \prime}}=\delta_{\varepsilon^{\prime}}^{\varepsilon}
$$

For further information about $S L(2)_{q}$ see [14-17].

We will say that the algebra $S L(2)_{q}$ acts on the algebra $A$ if it is defined by homomorphism of algebras:

$$
A \stackrel{\alpha}{\rightarrow} S L(2)_{q} \otimes A
$$

and the diagram

$$
A \stackrel{\alpha}{\rightarrow} S L(2)_{q} \otimes A \underset{\Delta \otimes \mathrm{id}}{\stackrel{\mathrm{id} \otimes \alpha}{\longrightarrow}} S L(2)_{q} \otimes S L(2)_{q} \otimes A
$$

is commutative (more precisely this is coaction).

If $A=\operatorname{End}(V)$ we will say that $S L(2)_{q}$ acts on $V$. In the classical limit $q \rightarrow 1$ generators $g_{\varepsilon^{\prime}}^{\varepsilon}$ become matrix elements of two-dimensional representation of $S L(2)$. In this limit the product

$$
g_{\varepsilon_{1}^{\prime}}^{\varepsilon_{1}} \cdots g_{\varepsilon_{n}^{\prime}}^{\varepsilon_{n}}
$$

are matrix elements of $n^{\text {th }}$ tensorial power of two-dimensional representation. This $2^{n}$-dimensional representation is reducible and decomposes into the sum of its irreducible components. In the quantum case this means that the matrix $(0.2)$ has block-diagonal structure (see $[15,17]$ for more details). $\left(\mathbb{C}^{2}\right)^{\otimes n}$ :

We are interested now only by singlets vectors $E^{\varepsilon_{1} \cdots \varepsilon_{n}}$ and covectors $E_{\varepsilon_{1} \cdots \varepsilon_{n}}^{\prime}$ in

$$
\begin{aligned}
& \sum_{\varepsilon_{1}^{\prime} \cdots \varepsilon_{n}^{\prime}} g_{\varepsilon_{1}^{\prime}}^{\varepsilon_{1}} \cdots g_{\varepsilon_{n}^{\prime}}^{\varepsilon_{n}} E^{\varepsilon_{1}^{\prime} \cdots \varepsilon_{n}^{\prime}}=E^{\varepsilon_{1} \cdots \varepsilon_{n},}, \\
& \sum_{\varepsilon_{1}^{\prime} \cdots \varepsilon_{n}^{\prime}}^{\varepsilon_{\varepsilon_{1}^{\prime}} \cdots \varepsilon_{n}^{\prime}} g_{\varepsilon_{1}}^{\varepsilon_{1}^{\prime} \cdots g_{\varepsilon_{n}}^{\varepsilon_{n}^{\prime}}}=E_{\varepsilon_{1} \cdots \varepsilon_{n}} .
\end{aligned}
$$


Let us denote the space of singlets in $\left(\mathbb{C}^{2}\right)^{\otimes n}$ as $\left(\left(\mathbb{C}^{2}\right)^{\otimes n}\right)_{\text {inv }}$. For generic $q\left(q^{n} \neq 1\right.$ for any $n \in \mathbb{N}$ ) this space is a span of vectors

$$
\begin{aligned}
E\left(l_{1} \cdots l_{n-2}\right)= & \sum_{\substack{\varepsilon_{1} \cdots \varepsilon_{n} \\
k_{1} \cdots k_{n-2}}}\left[\begin{array}{ccc}
\frac{1}{2} & \frac{1}{2} & l_{1} \\
\varepsilon_{1} & \varepsilon_{2} & k_{1}
\end{array}\right]_{q}\left[\begin{array}{ccc}
l_{1} & \frac{1}{2} & l_{2} \\
k_{1} & \varepsilon_{3} & k_{2}
\end{array}\right]_{q} \\
& \cdots\left[\begin{array}{ccc}
l_{n-2} & \frac{1}{2} & 0 \\
k_{n-2} & \varepsilon_{n} & 0
\end{array}\right]_{q} e_{\varepsilon_{1}} \otimes \cdots \otimes e_{\varepsilon_{n}}
\end{aligned}
$$

where

$$
\left[\begin{array}{lll}
j_{1} & \frac{1}{2} & j_{2} \\
m_{1} & \varepsilon & m_{2}
\end{array}\right]_{q}
$$

are $q-3 j$-symbols [17]:

$$
\begin{gathered}
{\left[\begin{array}{ccc}
j & \frac{1}{2} & j+\frac{1}{2} \\
m & \frac{1}{2} & m+\frac{1}{2}
\end{array}\right]_{q}=q^{(j-m) / 4} \sqrt{\frac{[j+1-m]}{[2 j+1]}}} \\
{\left[\begin{array}{ccc}
j & \frac{1}{2} & j+\frac{1}{2} \\
m & -\frac{1}{2} & m-\frac{1}{2}
\end{array}\right]_{q}=q^{(j+1+m) / 4} \sqrt{\frac{[j+1-m]}{[2 j+1]}},} \\
{\left[\begin{array}{ccc}
j & \frac{1}{2} & j-\frac{1}{2} \\
m & \frac{1}{2} & m+\frac{1}{2}
\end{array}\right]_{q}=q^{-(j+m+2) / 4} \sqrt{\frac{[j-m]}{[2 j+1]}}} \\
{\left[\begin{array}{ccc}
j & \frac{1}{2} & j-\frac{1}{2} \\
m & -\frac{1}{2} & m-\frac{1}{2}
\end{array}\right]_{q}=-q^{(j+1-m) / 4} \sqrt{\frac{[j-m]}{[2 j+1]}}}
\end{gathered}
$$

and

$$
e^{+}=\left(\begin{array}{l}
1 \\
0
\end{array}\right), \quad e^{-}=\left(\begin{array}{l}
0 \\
1
\end{array}\right)
$$

Dual vectors have the same form:

$$
\begin{aligned}
E^{\prime}\left(l_{1} \cdots l_{n-2}\right)= & \sum_{\varepsilon_{1} \cdots \varepsilon_{n}}\left[\begin{array}{ccc}
\frac{1}{2} & \frac{1}{2} & l_{1} \\
\varepsilon_{1} & \varepsilon_{2} & k_{1}
\end{array}\right]_{q}\left[\begin{array}{ccc}
l_{1} & \frac{1}{2} & l_{2} \\
k_{1} & \varepsilon_{2} & k_{2}
\end{array}\right]_{q} \\
& \cdots\left[\begin{array}{ccc}
l_{n-2} & \frac{1}{2} & 0 \\
k_{n-2} & \varepsilon_{n} & 0
\end{array}\right]_{q} e_{\varepsilon_{1}} \otimes \cdots \otimes e_{\varepsilon_{n}}
\end{aligned}
$$

where $\left\{e^{\varepsilon}\right\}$ is a dual basis for $\left\{e_{\varepsilon}\right\}$ in $\mathbb{C}^{2},\left(e^{\varepsilon}, e_{\varepsilon^{\prime}}\right)=\delta_{\varepsilon^{\prime}}^{\varepsilon}$. For generic $q$ these $q-3 j$-symbols satisfy orthogonality relations:

$$
\sum_{m, \varepsilon}\left[\begin{array}{ccc}
j & \frac{1}{2} & j^{\prime} \\
m & \varepsilon & m^{\prime}
\end{array}\right]_{q}\left[\begin{array}{ccc}
j & \frac{1}{2} & j^{\prime \prime} \\
m & \varepsilon & m^{\prime \prime}
\end{array}\right]_{q}=\delta_{j^{\prime} j^{\prime \prime}} \delta_{m^{\prime} m^{\prime \prime}}
$$

and therefore vectors $E^{\prime}\left(l_{1} \cdots l_{n-2}\right)$ are dual to $E\left(l_{1} \cdots l_{n-2}\right)$ under a standard scalar product in $\left(\mathbb{C}^{2}\right)^{\otimes n}$ :

$$
E^{\prime}\left(l_{1}^{\prime} \cdots l_{n-2}^{\prime}\right) E\left(l_{1} \cdots l_{n-2}\right)=\delta_{l_{1} l_{1}} \cdots \delta_{l_{n-2}^{\prime} l_{n-2}}
$$


Another situation we have when $q$ is a root of unity. Let $q^{r}=1$, then some of $q-3 j$-symbols become infinity. To have the finite vectors $E\left(l_{1}, \ldots, l_{n-1}\right)$ we should renormalize $q-3 j$-symbols. Put

$$
\left[\begin{array}{ccc}
j & \frac{1}{2} & j^{\prime} \\
m & \varepsilon & m+\varepsilon
\end{array}\right]=\left[\begin{array}{ccc}
j & \frac{1}{2} & j^{\prime} \\
m & \varepsilon & m+\varepsilon
\end{array}\right] \sqrt{[2 j+1]}
$$

These symbols are finite for any $q$. Therefore vectors are

$$
\begin{aligned}
\tilde{E}^{\prime}\left(l_{1} \cdots l_{n-2}\right) & =\prod_{\alpha=1}^{n-2} \sqrt{\left[2 l_{\alpha}+1\right]} E^{\prime}\left(l_{1} \cdots l_{n-2}\right), \\
\tilde{E}\left(l \cdots l_{n-2}\right) & =\prod_{\alpha-1}^{n-2} \sqrt{\left[2 l_{\alpha}+1\right]} E\left(l_{1} \cdots l_{n-2}\right),
\end{aligned}
$$

also finite for any $q$, and

$$
\tilde{E}^{\prime}\left(l_{1} \cdots l_{n-2}\right) E\left(l_{1}^{\prime} \cdots l_{n-2}^{\prime}\right)=\prod_{\alpha=1}^{n-2}\left[2 l_{\alpha}+1\right] \delta_{l_{\alpha}^{\prime} l_{\alpha}^{\prime}}
$$

It follows from these formulas that vectors $\tilde{E}\left(l_{1} \cdots l_{n-2}\right)$ has nonzero scalar products with $\tilde{E}^{\prime}\left(l_{1} \cdots l_{n-2}\right)$ only if the sequences $\left(l_{1} \cdots l_{n-2}\right)$ are restricted: $l_{i} \leqq r / 2-1$. So, from the subspace of $S L(2)_{q}$ invariant vectors in $\left(\mathbb{C}^{2}\right)^{\otimes 2}$ we can extract subspace of "good" vectors $E\left(l_{1} \cdots l_{n-2}\right)$ with restricted sequences $\left(l_{1} \cdots l_{n-2}\right)$. Other vectors in this subspace have a zero scalar square.

Therefore we have the decomposition of the space of one-dimensional subrepresentations in $\left(\mathbb{C}^{2}\right)^{\otimes n}$ on "bad" and "good" subspaces. The last one is formed by vectors $E\left(l_{1} \cdots l_{n-2}\right)$ with restricted sequences. The first one is formed by vectors having zero norm. A more precise description of "bad" subspace is given in [26].

Suppose $\alpha$ is an operator, acting in $\left(\mathbb{C}^{2}\right)^{\otimes n}$.

Let us suppose that if $0 \leqq l_{i} \leqq r / 2-1$ then

and

$$
\begin{aligned}
\alpha \cdot E\left(l_{1} \cdots l_{n-2}\right)= & \sum_{\operatorname{good}\left(l_{1} \cdots l_{n-2}^{\prime}\right)} \alpha_{l_{1} \cdots l_{n-2}}^{l_{1} \cdots l_{n-2}} E\left(l_{1}^{\prime} \cdots l_{n-2}^{\prime}\right) \\
& +\sum_{\operatorname{bad}\left(l_{1}^{\prime} \cdots l_{n-2}^{\prime}\right)}^{l_{l_{1}}^{\prime} \cdots l_{n-2}^{\prime}} \alpha_{l_{n-2}}^{l_{1} \cdots l_{n-2}} E\left(l_{1}^{\prime} \cdots l_{n-2}^{\prime}\right)
\end{aligned}
$$

$$
\alpha \cdot E\left(l_{1} \cdots l_{n-2}\right)=\sum_{\operatorname{bad}\left(l_{1}^{\prime} \cdots l_{n-2}^{\prime}\right)} \alpha_{l_{1} \cdots l_{n-2}}^{l_{1} \cdots l_{n-2}} E\left(l_{1}^{\prime} \cdots l_{n-2}^{\prime}\right)
$$

otherwise is the action of $\alpha$ on the basis in the space of one-dimensional subrepresentations of $S L(2)_{q}$ in $\left(\mathbb{C}^{2}\right)^{\otimes n}$. From orthogonality "good" and "bad" subspaces follows and because "bad" vectors has zero norm we have:

The important fact is that matrices

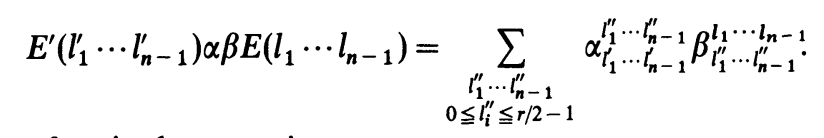

$$
\alpha_{i}=1 \otimes \cdots \otimes R_{(i, i+1)} \otimes \cdots \otimes 1
$$

in $\left(\mathbb{C}^{2}\right)_{\text {inv }}^{\otimes n}$ acts as $(0.2)(0.3)$. This can be proven by direct calculations. 


\section{1. $S L(2)_{q}$ Invariance of Sine-Gordon Models}

Let us return to the SG model. It has already been mentioned that the spectrum of SG contains solitons possessing an internal degree of freedom (solitonantisoliton) and (for $\xi>\pi$ ) their bound states - breathers. For the sake of simplicity let us consider the case $\xi>\pi$ when there are no breathers. Afterwards breathers can be easily taken into account because they are scalar with respect to the action of the quantum group described below.

For describing the space of states of a SG model, it is convenient to use Zamolodchikov-Faddeev operators $Z^{\varepsilon}(\beta), Z_{\varepsilon}^{*}(\beta)(\beta$ is rapidity, $\varepsilon= \pm 1)$. These operators satisfy the following commutation relations:

$$
\begin{aligned}
& Z^{\varepsilon_{1}}\left(\beta_{1}\right) Z^{\varepsilon_{2}}\left(\beta_{2}\right)=\sum_{\varepsilon_{1}^{\prime} \varepsilon_{2}^{\prime}} S_{\varepsilon_{1}^{\prime} \varepsilon_{2}^{\prime}}^{\varepsilon_{1}^{\prime} \varepsilon_{2}}\left(\beta_{1}-\beta_{2}\right) Z^{\varepsilon_{2}^{\prime}}\left(\beta_{2}\right) Z^{\varepsilon_{1}^{\prime}}\left(\beta_{1}\right), \\
& Z_{\varepsilon_{1}}^{*}\left(\beta_{1}\right) Z_{\varepsilon_{2}}^{*}\left(\beta_{2}\right)=\sum_{\varepsilon_{1}^{\prime} \varepsilon_{2}^{\prime}} S_{\varepsilon_{1} \varepsilon_{2}}^{\varepsilon_{1}^{\prime} \varepsilon_{2}^{\prime}}\left(\beta_{1}-\beta_{2}\right) Z_{\varepsilon_{2}^{\prime}}^{*}\left(\beta_{2}\right) Z_{\varepsilon_{1}^{\prime}}^{*}\left(\beta_{1}\right), \\
& Z^{\varepsilon_{1}}\left(\beta_{1}\right) Z_{\varepsilon_{2}}^{*}\left(\beta_{2}\right)=\sum_{\varepsilon_{1}^{\prime} \varepsilon_{2}^{\prime}}^{\ell_{\varepsilon_{2}}^{\prime}} Z_{2}^{*}\left(\beta_{2}\right) S_{\varepsilon_{1} \varepsilon_{2}}^{\varepsilon_{2} \varepsilon_{2}^{\prime}}\left(\beta_{2}-\beta_{1}\right) Z^{\varepsilon_{1}^{\prime}}\left(\beta_{1}\right)+\delta_{\varepsilon_{2}}^{\varepsilon_{1}} \delta\left(\beta_{1}-\beta_{2}\right),
\end{aligned}
$$

where $S(\beta)$ is the scattering matrix of solitons [4], which can be presented as an operator acting in $\mathbb{C}^{2} \otimes \mathbb{C}^{2}$ :

$$
\begin{aligned}
S_{12}(\beta)= & \frac{S_{0}(\beta)}{\operatorname{sh} \frac{\pi}{\xi}(\beta-\pi i)}\left\{\frac{1}{2}\left(\operatorname{sh} \frac{\pi}{\xi}(\beta-\pi i)-\operatorname{sh} \frac{\pi}{\xi} \beta\right) I\right. \\
& \left.+\frac{1}{2}\left(\operatorname{sh} \frac{\pi}{\xi}(\beta-\pi i)+\operatorname{sh} \frac{\pi}{\xi} \beta\right) \sigma_{1}^{3} \sigma_{2}^{3}-\operatorname{sh} \frac{\pi i}{\xi}\left(\sigma_{1}^{+} \sigma_{2}^{-}+\sigma_{1}^{-} \sigma_{2}^{+}\right)\right\}, \\
S_{0}(\beta)= & \exp \left(-i \int_{0}^{\infty} \frac{\sin k \beta \operatorname{sh}\left(\frac{\pi-\xi}{2}\right) k}{\left.k \operatorname{ch} \frac{\pi k}{2} \operatorname{sh} \frac{\xi k}{2} d k\right),}\right.
\end{aligned}
$$

where $\sigma_{i}^{3}, \sigma_{i}^{ \pm}$are Pauli matrices acting nontrivially in $i^{\text {th }}$ space $\left(\sigma_{1}^{a}=\left(\sigma^{a} \otimes I\right)\right.$, $\left.\sigma_{2}^{a}\left(I \otimes \sigma^{a}\right)\right)$. In what follows we shall use the notations of this kind and indices notations. We hope it will not give rise to any misunderstanding.

The operator $Z^{\varepsilon}(\beta)$ annihilates the vaccum $|0\rangle$ while the operator $Z_{\varepsilon}^{*}(\beta)$ creates one-soliton excitation. The space of states contains the vectors

$$
\left|\beta_{n} \cdots \beta_{1}\right\rangle_{\varepsilon_{1} \cdots \varepsilon_{1}}=Z_{\varepsilon_{n}}^{*}\left(\beta_{n}\right) \cdots Z_{\varepsilon_{1}}^{*}\left(\beta_{1}\right)|0\rangle
$$

and their linear combinations. The full set of "in" states consists of the vector (1.2) with $\beta_{n}>\cdots>\beta_{1}$, the full set of "out" states consists of the vectors (1.2) with $\beta_{n}<\beta_{n-1}<\cdots<\beta_{1}$. In the same manner we define the covectors

$$
\langle 0| Z^{\varepsilon_{1}}\left(\beta_{1}\right) \cdots Z^{\varepsilon_{n}}\left(\beta_{n}\right) \equiv{ }^{\varepsilon_{1} \cdots \varepsilon_{n}}\left\langle\beta_{1} \cdots \beta_{n}\right| .
$$

Now we define the action of quantum groups $G=S L(2)_{q}$ and $\bar{G}=S L(2)_{\bar{q}}$ $\left(q=\exp 2 \pi^{2} i / \xi\right)$ in the space of states. To put into agreement the definition of quantum group and the exact form of SG $S$-matrix we understand the square root 
$q^{1 / 2}$ in $(0.1)$ as $-\exp \left(\pi^{2} i / \xi\right)$. In the $n$-solitons sector these actions of $G$ and $\bar{G}\left(T_{R}, T_{L}\right)$ as slightly modified tensor powers:

$$
\begin{aligned}
& T_{R}(g)\left|\beta_{n} \cdots \beta_{1}\right\rangle_{\varepsilon_{n} \cdots \varepsilon_{1}}=\sum_{\varepsilon_{1}^{\prime} \cdots \varepsilon_{n}^{\prime}}\left|\beta_{n} \cdots \beta_{1}\right\rangle_{\varepsilon_{n}^{\prime} \cdots \varepsilon_{1}^{\prime}} g_{\varepsilon_{n}}^{\varepsilon_{n}^{\prime} \cdots} g_{\varepsilon_{1}}^{\varepsilon_{1}^{\prime}} \exp \left(\frac{\pi}{2 \xi} \sum \beta_{j}\left(\varepsilon_{j}-\varepsilon_{j}^{\prime}\right)\right), \\
& T_{L}(\bar{g})\left|\beta_{n} \cdots \beta_{1}\right\rangle_{\varepsilon_{n} \cdots \varepsilon_{1}}=\sum_{\varepsilon_{1}^{\prime} \cdots \varepsilon_{n}^{\prime}}\left|\beta_{n} \cdots \beta_{1}\right\rangle_{\varepsilon_{n}^{\prime} \cdots \varepsilon_{1}^{\prime}} \bar{g}_{\varepsilon_{n}}^{\varepsilon_{n}^{\prime}} \cdots \bar{g}_{\varepsilon_{1}}^{\varepsilon_{1}^{\prime}} \exp \left(-\frac{\pi}{2 \xi} \sum \beta_{j}\left(\varepsilon_{j}-\varepsilon_{j}^{\prime}\right)\right), \\
& \varepsilon_{1} \cdots \varepsilon_{n}\left\langle\beta_{1} \cdots \beta_{n}\right| T_{R}(g)=\sum_{\varepsilon_{1}^{\prime} \cdots \varepsilon_{n}^{\prime}} \exp \left(-\frac{\pi}{2 \xi} \sum \beta_{j}\left(\varepsilon_{j}-\varepsilon_{j}^{\prime}\right)\right) g_{\varepsilon_{1}^{\prime}}^{\varepsilon_{1}} \cdots g_{\varepsilon_{n}^{\prime}}^{\varepsilon_{n} \varepsilon_{1}^{\prime} \cdots \varepsilon_{n}^{\prime}}\left\langle\beta_{1} \cdots \beta_{n}\right|, \\
& \varepsilon_{1} \cdots \varepsilon_{n}\left\langle\beta_{1} \cdots \beta_{n}\right| T_{L}(\bar{g})=\sum_{\varepsilon_{1}^{\prime} \cdots \varepsilon_{n}^{\prime}} \exp \left(\frac{\pi}{2 \xi} \sum \beta_{j}\left(\varepsilon_{j}-\varepsilon_{j}^{\prime}\right)\right) \bar{g}_{\varepsilon_{1}^{\prime}}^{\varepsilon_{1}} \cdots \bar{g}_{\varepsilon_{n}}^{\varepsilon_{n} n_{1}^{\prime} \cdots \varepsilon_{n}^{\prime}}\left\langle\beta_{1} \cdots \beta_{n}\right| .
\end{aligned}
$$

Now it is necessary to make sure in self-consistency of these definitions. Really, we can permute the particles $\beta_{i}, \beta_{i+1}$ and then apply $T_{R}\left(T_{L}\right)$ or first apply $T_{R}\left(T_{L}\right)$ and then permute the particles $\beta_{i}, \beta_{i+1}$. The result should be the same which means that the following identity should hold:

$$
\begin{aligned}
& \tilde{S}_{12}\left(\beta_{1}-\beta_{2}\right) g_{1} g_{2}=g_{2} g_{1} \tilde{S}_{12}\left(\beta_{1}-\beta_{2}\right), \\
& \tilde{\tilde{S}}_{12}\left(\beta_{1}-\beta_{2}\right) \bar{g}_{1} \bar{g}_{2}=\bar{g}_{2} \bar{g}_{1} \tilde{\widetilde{S}}_{12}\left(\beta_{1}-\beta_{2}\right),
\end{aligned}
$$

where

$$
\begin{aligned}
\tilde{S}_{12}\left(\beta_{1}-\beta_{2}\right)= & S_{0}^{-1}\left(\beta_{1}-\beta_{2}\right) \exp \frac{\pi}{2 \xi}\left(\sigma_{1}^{3} \beta_{1}+\sigma_{2}^{3} \beta_{2}\right) S_{12}\left(\beta_{1}-\beta_{2}\right) \\
& \cdot \exp \left(-\frac{\pi}{2 \xi}\left(\sigma_{1}^{3} \beta_{1}+\sigma_{2}^{3} \beta_{2}\right)\right) \\
\tilde{S}_{12}\left(\beta_{1}-\beta_{2}\right)= & S_{0}^{-1}\left(\beta_{1}-\beta_{2}\right) \exp \left(-\frac{\pi}{2 \xi}\left(\sigma_{1}^{3} \beta_{1}+\sigma_{2}^{3} \beta_{2}\right)\right) S_{12}\left(\beta_{1}-\beta_{2}\right) \\
& \cdot \exp \frac{\pi}{2 \xi}\left(\sigma_{1}^{3} \beta_{1}+\sigma_{2}^{3} \beta_{2}\right),
\end{aligned}
$$

$\left(\tilde{S}\right.$ and $\tilde{\widetilde{S}}$ are divided by $S_{0}$ because this multiplier can be omitted in (1.4).) The validity of the Eqs. (1.4) is a consequence of the following representations for $\tilde{S}, \tilde{\widetilde{S}}$ :

$$
\begin{aligned}
& \tilde{S}_{12}(\beta)=\frac{\left(e^{-(\pi / \xi) \beta} R_{12}(q)-e^{(\pi / \xi) \beta}\left(R_{21}(q)\right)^{-1}\right)}{2 \operatorname{sh} \frac{\pi}{\xi}(\beta-\pi i)}, \\
& \tilde{S}_{12}(\beta)=\frac{\left(e^{-(\pi / \xi) \beta}\left(R_{21}(\bar{q})\right)^{-1}-e^{(\pi / \xi) \beta} R_{12}(\bar{q})\right)}{2 \operatorname{sh} \frac{\pi}{\xi}(\beta-\pi i)} .
\end{aligned}
$$

These representations can be easily obtained from (1.1). 
From the physical point of view the fact of self-consistency of the definitions (1.3) means that $T_{R}$ and $T_{L}$ commute with the $S$-matrix, i.e., they really define some symmetry of the theory. The problem of consistency of this symmetry with the local description will be discussed below. Now we would like to notice one important property of $T_{R}$ and $T_{L}$ : they are conjugated via the operation of charge conjugation

$$
C T_{R}(g) C^{-1}=T_{L}\left(\sigma^{1} g \sigma^{1}\right)
$$

The charge conjugation is defined in SG as follows:

$$
C Z_{\varepsilon}^{*}(\beta) C^{-1}=Z_{-\varepsilon}^{*}(\beta), \quad C|0\rangle=|0\rangle .
$$

The action (1.3) of quantum groups $S L(2)_{q}, S L(2)_{\bar{q}}$ is equivalent to the following action of these quantum groups on the algebra (1.0):

$$
\begin{aligned}
& \alpha_{L}\left(Z_{\varepsilon}^{*}(\beta)\right)=\sum_{\varepsilon^{\prime}} Z_{\varepsilon^{\prime}}^{*}(\beta) \otimes g_{\varepsilon}^{\varepsilon^{\prime}} \exp \left(\frac{\pi}{2 \xi} \beta\left(\varepsilon-\varepsilon^{\prime}\right)\right), \\
& \alpha_{L}\left(Z^{\varepsilon}(\beta)\right)=\sum_{\varepsilon^{\prime}} Z^{\varepsilon^{\prime}}(\beta) \otimes \bar{g}_{\varepsilon^{\prime}}^{\varepsilon} \exp \left(-\frac{\pi}{2 \xi} \beta\left(\varepsilon-\varepsilon^{\prime}\right)\right), \\
& \alpha_{R}\left(Z_{\varepsilon}^{*}(\beta)\right)=\sum_{\varepsilon^{\prime}} Z_{\varepsilon^{\prime}}^{*}(\beta) \otimes g_{\varepsilon}^{\varepsilon^{\prime}} \exp \left(-\frac{\pi}{2 \xi} \beta\left(\varepsilon-\varepsilon^{\prime}\right)\right), \\
& \alpha_{R}\left(Z^{\varepsilon}(\beta)\right)=\sum_{\varepsilon^{\prime}} Z^{\varepsilon^{\prime}}(\beta) \otimes \bar{g}_{\varepsilon^{\prime}}^{\varepsilon} \exp \left(\frac{\pi}{2 \xi} \beta\left(\varepsilon-\varepsilon^{\prime}\right)\right) .
\end{aligned}
$$

For $\xi \rightarrow \infty$ the parameter $q \rightarrow 1$ and both $G$ and $\bar{G}$ turn into the classical group $S L(2)$. The model SG in this limit becomes an $S U(2)$-invariant Thirring model [8]. The actions $T_{L}$ and $T_{R}$ turn into the usual action of an isotopic group.

There is nothing especially surprising in the construction described above. Really, the very definition of quantum groups appeared from the study of trigonometrical solutions of Yang-Baxter equations. SG $S$-matrix is just one of these solutions. The invariance of the $S$-matrix model $S L(2)_{q}$ is a rather evident fact. The main problem is to understand the transformation properties of local operators under the actions $T_{R}, T_{L}$. One of the possible situations is that the local operator appears to be a sum of nonlocal operators which transform according to different irreducible representations of $G(\bar{G})$. In that case one has to conclude that on-shell symmetry is occasional and does not correspond to any off-shell symmetry. Fortunately, it is not the case in the situation under consideration. There are local operators which transform under irreducible representations of $G, \bar{G}$. In this paper we shall show in particular that there are operators invariant under $T_{R}\left(T_{L}\right)$. These operators create two subalgebras of the full operator algebra of the theory $A_{R}^{\text {inv }}$ and $A_{L}^{\text {inv. }}$ :

$$
\begin{array}{llll}
T_{R}(g) O(x)=O(x) T_{R}(g) & \text { if } & O \in A_{R}^{\text {inv }} \\
T_{L}(g) O(x)=O(x) T_{L}(g) & \text { if } & O \in A_{L}^{\text {inv }}
\end{array} .
$$

Equation (1.5) implies that

$$
A_{\mathrm{R}}^{\mathrm{inv}}=C A_{\mathrm{L}}^{\mathrm{inv}} C^{-1} .
$$


The natural way of describing local operators in massive theory is fixing their matrix elements in the space of states. In the integrable case these matrix elements satisfy the system of Eqs. [18-19] which allows exact calculation in many significant cases.

Consider some local operator $O(x)$. We call formfactors the following matrix elements of $O$ :

$$
f\left(\beta_{1} \cdots \beta_{n}\right)_{\varepsilon_{1} \cdots \varepsilon_{n}}=\left\langle 0|O(0)| \beta_{n} \cdots \beta_{1}\right\rangle_{\varepsilon_{n} \cdots \beta_{1}} .
$$

The matrix elements of general type can be expressed in terms of formfactors:

$$
\varepsilon_{1}^{\prime} \cdots \varepsilon_{m}^{\prime}\left\langle\alpha_{1} \cdots \alpha_{m}|O(0)| \beta_{k} \cdots \beta_{1}\right\rangle_{\varepsilon_{k} \cdots \varepsilon_{1}}=f\left(\alpha_{m} \cdots \alpha_{1} \mid \beta_{1} \cdots \beta_{k}\right)_{\varepsilon_{1} \cdots \varepsilon_{k}}^{s_{1}^{\prime} \cdots \varepsilon_{1}^{\prime}}+\cdots
$$

where

$$
f\left(\alpha_{m} \cdots \alpha_{1} \mid \beta_{1} \cdots \beta_{k}\right)_{\varepsilon_{1} \cdots \varepsilon_{k}}^{\varepsilon_{m}^{\prime} \cdots \varepsilon_{1}^{\prime}}=\sum_{\varepsilon_{m}^{\prime \prime} \varepsilon_{1}^{\prime \prime}} c^{\varepsilon_{m}^{\prime} \varepsilon_{m}^{\prime \prime}} c^{\varepsilon_{1}^{\prime} \varepsilon_{1}^{\prime \prime}} f\left(\beta_{1} \cdots \beta_{k}, \alpha_{m}+\pi_{i}, \ldots, \alpha_{1}+\pi_{i}\right)_{\varepsilon_{1} \cdots \varepsilon_{k} \varepsilon_{m}^{\prime \prime} \cdots \varepsilon_{1}^{\prime \prime}},
$$

$c=\sigma^{1}$, dots in (1.6) mean the term containing $\delta$-functions which are not very essential here.

We want to find $T_{R}$-invariant operators. Their matrix elements should satisfy the equations:

$$
\begin{aligned}
& \left.\sum_{\varepsilon_{1}^{\prime \prime} \cdots \varepsilon_{n}^{\prime \prime}} g_{\varepsilon_{1}^{\varepsilon_{1}^{\prime}}}^{\varepsilon_{1}^{\prime}} \cdots g_{\varepsilon_{k}^{\prime \prime}}^{\varepsilon_{k}^{\prime}} \exp \left(-\frac{\pi}{2 \xi} \sum \alpha_{i}\left(\varepsilon_{i}^{\prime}-\varepsilon_{i}^{\prime \prime}\right)\right)\right)^{\varepsilon_{1}^{\prime \prime} \cdots \varepsilon_{k}^{\prime \prime}}\left\langle\alpha_{1} \cdots \alpha_{k}|O(0)| \beta_{m} \cdots \beta_{1}\right\rangle_{\varepsilon_{m} \cdots \varepsilon_{1}} \\
& =\sum_{\varepsilon_{1}^{\prime \prime \prime} \cdots \varepsilon_{m}^{\prime \prime}} \varepsilon_{1}^{\prime} \cdots \varepsilon_{k}^{\prime}\left\langle\alpha_{1} \cdots \alpha_{k}|O(0)| \beta_{m} \cdots \beta_{1}\right\rangle_{\varepsilon_{m}^{\prime \prime \prime} \cdots \varepsilon_{1}^{\prime \prime}} \underbrace{\varepsilon_{m}^{\prime \prime \prime} \cdots}_{\varepsilon_{m}} g_{\varepsilon_{1}}^{\varepsilon_{1}^{\prime \prime \prime}} \exp \left(\frac{\pi}{2 \xi} \sum \alpha_{i}\left(\varepsilon_{i}-\varepsilon_{i}^{\prime \prime \prime}\right)\right) \text {. }
\end{aligned}
$$

Equations (1.6),(1.7) together with the identities

$$
s(g)=\alpha g^{t} \alpha, \quad \alpha=\sigma^{1} \exp \left(\frac{\pi^{2} i}{2 \xi} \sigma_{3}\right), \quad\left\{\sigma^{1}, \sigma^{3}\right\}=0, \quad g s(g)=s(g) g=1
$$

imply that the sufficient condition of $T_{R}$ invariance is the following identity for formfactors:

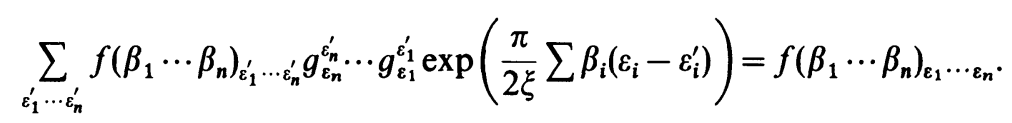

In other notations this identity can be written as

$$
f\left(\beta_{1} \cdots \beta_{n}\right)_{1} \cdots n \exp \left(-\frac{\pi}{2 \xi} \sum \sigma_{i}^{3} \beta_{i}\right) g_{n} \cdots g_{1} \exp \left(\frac{\pi}{2 \xi} \sum \sigma_{i}^{3} \beta_{i}\right)=f\left(\beta_{1} \cdots \beta_{n}\right)_{1} \cdots n,
$$

here $f\left(\beta_{1} \cdots \beta_{n}\right)_{1} \cdots n$ means covector from $\left(\mathbb{C}^{2}\right)^{\otimes n}$.

In the papers [20] the formfactors were obtained for the operators $\varphi, T_{\mu v}$, $\exp ( \pm i \sqrt{\gamma} \varphi), \exp ( \pm i \sqrt{\gamma} \varphi / 2)$. We shall show that the operators $\exp (i \sqrt{\gamma} \varphi)$, $\exp (i \sqrt{\gamma} \varphi / 2)$ and modified energy-momentum tensor

$$
\tilde{T}_{\mu \nu}=T_{\mu \nu}+i 2^{-5 / 2} \frac{\sqrt{\gamma}}{\xi} \varepsilon_{\mu \mu^{\prime}} \varepsilon_{v v^{\prime}} \partial_{\mu^{\prime}} \partial_{v^{\prime}} \varphi
$$


whose trace is equal to [9]:

$$
\tilde{T}_{\mu \mu}=\frac{1}{2} M^{2} \operatorname{tg} \frac{\xi}{2} \exp (i \sqrt{\gamma} \varphi)
$$

( $M$ is soliton mass) are $T_{R}$-invariant.

Consider for example the operator $\exp (i \sqrt{\gamma} \varphi / 2)$. In the paper [20] the formfactors are shown to be

$$
\begin{aligned}
f\left(\beta_{1} \cdots \beta_{2 n}\right)_{1} \cdots 2 n & =c^{n} \prod_{i<j} \zeta\left(\beta_{i}-\beta_{j}\right) \int_{-\infty}^{\infty} d \alpha_{1} \cdots \int_{-\infty}^{\infty} d \alpha_{n-1} \prod_{i=1}^{n-1} \prod_{j=1}^{2 n} \varphi\left(\alpha_{i}-\beta_{j}\right) \prod_{i<j}^{n-1} \operatorname{sh}\left(\alpha_{i}-\alpha_{j}\right) \\
& \cdot \exp \left(-\frac{\pi}{\xi}(n-2) \sum \alpha_{i}+\frac{\pi}{\xi} \sum \beta_{j}\right) \\
& \cdot F\left(\exp \frac{2 \pi}{\xi} \alpha_{1} \cdots \exp \frac{2 \pi}{\xi} \alpha_{n-1} \mid \exp \frac{2 \pi}{\xi} \beta_{1} \cdots \exp \frac{2 \pi}{\xi} \beta_{2 n}\right)_{1 \cdots 2 n} \exp \left(\frac{\pi}{2 \xi} \sum \beta_{j} \sigma_{j}^{3}\right),
\end{aligned}
$$

where

$$
\begin{aligned}
& \varphi(\alpha)=c_{1} \exp \left(-\int_{0}^{\infty} \frac{2 \sin ^{2} \frac{\beta k}{2} \operatorname{sh}\left(\frac{\pi}{2}+\frac{\xi}{2}\right) k}{k \operatorname{sh} \frac{\xi k}{2} \operatorname{sh} \pi k} d k\right), \\
& \zeta(\beta)=c_{2} \operatorname{sh} \frac{\beta}{2} \exp \left(\int \frac{\sin ^{2} \frac{1}{2}(\beta+\pi i) k \operatorname{sh}\left(\frac{\pi-\xi}{2}\right) k}{k \operatorname{sh} \frac{\xi k}{2} \operatorname{sh} \pi k \operatorname{ch} \frac{\pi k}{2}} d k\right),
\end{aligned}
$$

$c, c_{1}, c_{2}$ are certain constants, $\int$ means a special regularization of the integral [20]. The most essential for our goal object is the function $F$. Let us discuss its property.

The function $F\left(a_{1} \cdots a_{n-1} \mid b_{1} \cdots b_{2 n}\right)_{1} \cdots 2 n$ is tensor-valued rational function of all its variables. It is a homogeneous function of all its variables of total degree $((n-1)(n-2) / 2)-n . F\left(a_{1} \cdots a_{n-1} \mid b_{1} \cdots b_{2 n}\right)$ is an antisymmetrical function of $a_{1} \cdots a_{n-1}$, its symmetry property with respect to $b_{1} \cdots b_{2 n}$ are given by the relation:

$$
F\left(a_{1} \cdots a_{n-1} \mid b_{1} \cdots b_{2 n}\right)_{1} \cdots 2 n \tilde{S}_{i i+1}\left(b_{i} b_{i+1}=F\left(a_{1} \cdots a_{n-1} \mid b_{1} \cdots b_{i+1} b_{i} \cdots b_{2 n}\right)\right.
$$

where

$$
\tilde{S}\left(b_{1}, b_{2}\right)=\tilde{S}\left(\beta_{1}-\beta_{2}\right)=\frac{b_{2} R_{12}(q)-b_{1}\left(R_{21}(q)\right)^{-1}}{b_{1} q^{-1 / 2}-b_{2} q^{1 / 2}}
$$

The function $F\left(a_{1} \cdots a_{n-1} \mid b_{1} \cdots b_{2 n}\right)_{1} \cdots n$ has no other singularities but simple poles 
at the points $b_{j}=b_{i} q$ for $j>i$. Its residues at these poles are

$$
\begin{aligned}
\underset{b_{i}=b_{j}, j>i}{\text { res }} & F\left(a^{1} \cdots a_{n-1} \mid b_{1} \cdots b_{2 n}\right)_{1} \cdots n \\
= & \sum_{k=1}^{n-1}(-1)^{k} F\left(a_{1} \cdots \hat{a}_{k} \cdots a_{n-1} \mid b_{1} \cdots \hat{b}_{i} \cdots \hat{b}_{j} \cdots b_{2 n}\right)_{1 \cdots \hat{i} \cdots j \cdots 2 n} \\
& \otimes \operatorname{singl}_{j, i}\left\{\prod_{p \neq i, j}\left(a_{k}+q^{1 / 2} b_{p}\right)-q^{2(n-1)} \prod_{p \neq i, j}\left(a_{k}+q^{-1 / 2} b_{p}\right)\right\} x_{k}^{-1} \\
& \cdot \prod_{p=1}^{n-1}\left(x_{p}+b_{i} q^{1 / 2}\right) \prod_{l \leqq i} \frac{1}{b_{l}-b_{i}} \prod_{l>i}\left(b_{l} q^{-1 / 2}-b_{i} q^{1 / 2}\right) \\
& \cdot \tilde{S}_{j-1, i}\left(b_{j-1}, b_{i}\right) \cdots \tilde{S}_{i+1, i}\left(b_{i+1}, b_{i}\right),
\end{aligned}
$$

where singl ${ }_{j, i}$ is singlet vector in the tensor product of $j^{\text {th }}$ and spaces:

$$
\left(q^{1 / 4} e_{1} \otimes e_{-1}-q^{-1 / 4} e_{-1} \otimes e_{1}\right) \text {. }
$$

Being antisymmetrical with respect to $a_{1} \cdots a_{n-1}$ the function $F\left(a_{1} \cdots a_{n-1} \mid b_{1} \cdots b_{2 n}\right)$ must contain the multiplier $\prod_{i<j}\left(a_{i}-a_{j}\right)$, hence its nontrivial part has total degree $(-n)$. That is why the function is defined completely by the requirement that it has no singularities but the poles $b_{j}=b_{i} q$ and the residues are given by (1.11). So, the Eq. (1.11) provides complete recurrent procedure for the calculation of $F$. An exact formula for the function $F$ can be written [15] but it is not important here.

The requirement of $T_{R}$ invariant $\exp (i \sqrt{\gamma} \varphi / 2)(1.8)$ is satisfied if $F$ satisfies the equation

$$
F\left(\beta_{1} \cdots \beta_{2 n}\right)_{1} \cdots n g_{2 n} \cdots g_{1}=F\left(\beta_{1} \cdots \beta_{2 n}\right)_{1} \cdots 2 n,
$$

which means that the vector $F$ lies in the $S L(2)_{q}$ spin $O$ subspaces of $\left(\mathbb{C}^{2}\right)^{\otimes 2 n}$. Look at Eq. (1.11). Having in mind that

$$
\begin{gathered}
\operatorname{singl}_{j i} g_{j} g_{i}=\left(g_{1}^{1} g_{2}^{2}-q^{1 / 2} g_{1}^{2} g_{2}^{1}\right) \operatorname{singl}_{j i}=\operatorname{singl}_{j i}, \\
\tilde{S}_{k i}\left(b_{k}, b_{i}\right) g_{k} g_{i}=g_{i} g_{k} \widetilde{S}_{k i}\left(b_{k}, b_{i}\right)
\end{gathered}
$$

one makes sure that the functions

$$
F\left(\beta_{1} \cdots \beta_{2 n}\right)_{1} \cdots 2 n g_{2 n} \cdots g_{1}
$$

satisfy the same system of recurrent relations as the functions $F\left(\beta_{1} \cdots \beta_{2 n}\right)$. The initial conditions are the same because

$$
F\left(\beta_{1}, \beta_{2}\right)_{12}=\operatorname{singl}_{21} .
$$

Hence the Eq. (1.12) holds for all $n$ by induction.

Thus we prove $T_{R}$ invariance of $\exp (i \sqrt{\gamma} \varphi / 2)$. Formfactors of the operators $\exp (i \sqrt{\gamma} \varphi), \tilde{T}_{\mu \nu}$ are given by the formulas similar to that for $\exp (i \sqrt{\gamma} \varphi / 2)$. The only difference is that additional multipliers $\exp \left( \pm\left(\sum \alpha_{i}-1 / 2 \sum \beta_{j}\right)\right)$ appear under 
the integral. Evidently this difference does not affect the fact of $T_{R}$ invariance because it is based only on the Eq. (1.12) for the function $F$.

We have proved $T_{R^{-}}$invariance of the operators $\tilde{T}_{\mu \nu}, \exp (i \sqrt{\gamma} \varphi), \exp (i \sqrt{\gamma} \varphi / 2)$. If the operator $O(x)$ is $T_{R}$-invariant then the operator $C O(x) C^{-1}$ is $T_{L}$-invariant due to (1.5). That is why the operators $\exp (-(i \sqrt{\gamma} \varphi / 2)), \exp (i \sqrt{\gamma} \varphi), \tilde{T}_{\mu \nu}^{*}$ are $T_{L}$-invariant, this fact can be proven directly using the formulas for their formfactors. It can be shown also that the operators $\exp (-(i \sqrt{\gamma} \varphi / 2)), \exp (-i \sqrt{\gamma} \varphi)$, $\tilde{T}_{\mu \nu}^{*}$ possess extremely bad properties with respect to $T_{R} .:$ they can be presented as infinite sum of nonlocal parts transforming under a different irreducible representation of $S L(2)_{q}$.

Evidently the algebra $A_{R}^{\text {inv }}$ is closed. Certainly it should contain besides the operators mentioned above the following ones: $\exp (i k \sqrt{\gamma} \varphi / 2)$ for arbitrary integer $k \geqq$. We do not know formulas for the formfactors of these operators but it is not a great problem to get them. Besides, the algebra $A_{R}^{\text {inv }}$ contains together with the operator $O(x)$ the operators $\left[I_{s}, O(x)\right],\left[I_{-s}, O(x)\right]$, where $I_{s}, I_{-s}$ are the local conservation laws of SG of spin $s(s \equiv 1(\bmod 2))$. Formfactors of these operators are expressed in terms of formfactors of $O$ as follows:

$$
\text { const }\left(\sum \exp \left( \pm s \beta_{j}\right)\right) f\left(\beta_{1} \cdots \beta_{1} \cdots \beta_{2 n}\right)_{\varepsilon_{1} \cdots \varepsilon_{2 n}} \text {. }
$$

Is this list of operators complete? It would be very interesting to answer this question, but we cannot do it at the present time.

\section{Reductions of Sine-Gordon for Rational $\boldsymbol{\xi} / \boldsymbol{\pi}$}

Consider a Green function of several $T_{R}$ invariant operators:

$$
\left\langle 0\left|O_{1}\left(x_{1}\right) \cdots O_{n}\left(x_{n}\right)\right| 0\right\rangle \text {. }
$$

Evidently it contains as intermediate states only the following combinations of pure SG states (for convenience we introduce $l_{1} \equiv \frac{1}{2}$ ):

$$
\begin{gathered}
\sum_{\varepsilon_{1} \cdots \varepsilon_{n}}\left|\beta_{n} \cdots \beta_{1}\right\rangle_{\varepsilon_{n} \cdots \varepsilon_{1}} E^{\varepsilon_{n} \cdots \varepsilon_{1}}\left(l_{n-1} \cdots l_{1}\right) \exp \left(-\frac{\pi}{2 \xi} \sum e_{j} \beta_{j}\right) \equiv\left|\beta_{n} l_{n-l} \beta_{n-1} \cdots l_{1} \beta_{1}\right\rangle \\
\sum_{\varepsilon_{1} \cdots \varepsilon_{n}} E_{\varepsilon_{1} \cdots \varepsilon_{n}}^{\prime}\left(l_{1} \cdots l_{n-1}\right) \exp \left(\frac{\pi}{2 \xi} \sum \varepsilon_{j} \beta_{j}\right)^{\varepsilon_{1} \cdots \varepsilon_{n}}\left\langle\beta_{1} \cdots \beta_{n}\right|=\left\langle\beta_{1} l_{1} \cdots l_{n-1} \beta_{n}\right| .
\end{gathered}
$$

The vectors $E, E^{\prime}$ were defined in Sect. 0 .

It has been said in Sect. 0 that the properties of $E, E^{\prime}$ defined essentially on the nature of $q$. If $(\pi / \xi)$ is a rational number then one has

$$
E^{\prime}\left(l_{1} \cdots l_{n-1}\right) E\left(l_{n-1}^{\prime} \cdots l_{1}^{\prime}\right)=\delta_{l^{\prime} l_{1}^{\prime}} \cdots \delta_{l_{n-1} l_{n-1}^{\prime}} .
$$

If $\xi=\left(r / r_{1}\right) \pi$ which means that $q^{r}=1$ then the states with intermediate spins greater than $(r-2) / 2$ appear to be "orthogonal" to themselves:

$$
E^{\prime}\left(l_{1} \cdots l_{n-1}\right) E\left(l_{n-1}^{\prime} \cdots l_{1}^{\prime}\right)=\chi_{r}\left(l_{1}\right) \cdots \chi_{r}\left(l_{n-1}\right) \delta_{l_{1} l_{1}^{\prime}} \cdots \delta_{l_{n-1} l_{n-1}^{\prime}},
$$


where

$$
\chi_{r}(a)=\left\{\begin{array}{lll}
1 & \text { if } & 0 \leqq a \leqq \frac{r-2}{2} \\
0 & \text { if } & a>\frac{r-2}{2}
\end{array}\right.
$$

Let us denote by $P_{r}\left(\tilde{P}_{r}\right)$ the operator which projects the space of states of SG (dual space) onto the subspace spanned on the vectors $(2.1)((2.2))$ with $l_{1} \leqq(r-2) / 2$. The above reasonings imply that for $\xi=\pi\left(r / r_{1}\right)$ the following identity holds for the Green functions of $T_{R}$ invariant operators:

$$
\left\langle O_{1}\left(x_{1}\right) \cdots O_{n}\left(x_{n}\right)\right\rangle=\left\langle\hat{O}_{1}\left(x_{1}\right) \cdots \hat{O}_{n}\left(x_{n}\right)\right\rangle,
$$

where

$$
\hat{O}(x)=\tilde{P}_{r}\left(O(x) P_{r} .\right.
$$

The identity (2.3) means that the operators $\hat{O}_{i}(x)$ are local and mutually local. However, they are not mutually local with those SG operators which are not $T_{R}$-invariant. Thus we deal with certain restrictions of SG theory which is a consequence of special properties of $S L(2)_{q}$ for $q^{r}=1$.

The next important problem is the possibility of physical interpretation of reduced models (we denote them by $\mathrm{RSG}\left(r / r_{1}\right)$ following [9]). The space of states of the reduced model is $H_{r}=P_{r} H$ ( $H$ is the space of states of SG). It is not clear how to introduce in $H_{r}$ the structure of Hilbert space. The structure induced from SG is not suitable because the space conjugated to $H_{r}$ does not coincide with $H_{r}^{\prime}$. Notice that in all the reasonings concerned with locality $[18,19]$ the Hilbert structure is not involved. In fact the space spanned on the vectors

$$
\left|\beta_{n} \cdots \beta_{1}\right\rangle_{\varepsilon_{r} \cdots \varepsilon_{1}}
$$

and that spanned on

$$
\varepsilon_{1} \cdots \varepsilon_{n}\left\langle\beta_{1} \cdots \beta_{n}\right|
$$

are used as dual linear spaces. The Hilbert structure of SG space of states which require that

$$
\left(\left|\beta_{n} \cdots \beta_{1}\right\rangle_{\varepsilon_{n} \cdots \varepsilon_{1}}\right)^{*}={ }^{\varepsilon_{1} \cdots \varepsilon_{n}}\left\langle\beta_{1} \cdots \beta_{n}\right|
$$

is from this point of view, an external condition needed for the correct physical interpretation. SG Hilbert structure ensures for example the relations:

$$
P_{\mu}=P_{\mu}^{*}, T_{\mu \nu}^{*}=T_{\mu \nu},\left(\exp \left(i \frac{\sqrt{\gamma}}{2} \varphi\right)\right)^{*}=\exp \left(-i \frac{\sqrt{\gamma}}{2} \varphi\right)
$$

etc.

In the reduced model we introduce another Hilbert structure. Namely, we require that

$$
\left|\beta_{r} l_{n-1} \beta_{n-1} \cdots l_{1} \beta_{1}\right\rangle^{\star}=\left\langle\beta_{1} l_{1} \cdots l_{n-1} \beta_{r}\right|
$$


The symbol $\star$ in this formula has another meaning than in SG. After the new Hilbert structure is introduced we have evidently $H_{r}^{\prime}=H_{r}^{*}$. The operators $\hat{O}(x)$ acting in $H_{r}$ are local and their Green functions coincide with SG ones. It is not clear, however, that the reduced model with the Hilbert structure (2.9) allows interpretation in terms of particles. Let us discuss the problem in more detail.

The most important operator in $\operatorname{RSG}\left(r / r_{1}\right)$ is the energy-momentum tensor

$$
\mathscr{T}_{\mu \nu}=\tilde{T}_{\mu \nu}
$$

The operator

$$
P_{\mu}=\int T_{\mu 0}\left(x_{1}\right) d x_{1}
$$

coincides with the restriction of SG energy-momentum into $H_{r}$, i.e., its eigenvalues are:

$$
\begin{aligned}
P_{\mu}\left|\beta_{n} l_{n-1} \cdots l_{1} \beta_{1}\right\rangle & =\sum_{j=1}^{n} M\left(e^{\beta_{j}}+(-1)^{\mu} e^{-\beta_{j}}\right)\left|\beta_{n} l_{n-1} \cdots l_{1} \beta_{1}\right\rangle, \\
\left\langle\beta_{1} l_{1} \cdots \beta_{n}\right| P_{\mu} & =\sum_{j=1}^{n} M\left(e^{\beta_{j}}+(-1)^{\mu} e^{-\beta_{j}}\right)\left\langle\beta_{1} l_{1} \cdots \beta_{n}\right| .
\end{aligned}
$$

Thus energy-momentum operator is selfadjoint in new Hilbert structure and energy is positive.

Consider the $S$-matrix which is the restriction of SG on $H_{r}$. Multiparticle scattering can be reduced to two-particles processes. Two-particles amplitudes are defined by:

$$
\sum_{l_{k}}\left|\cdots l_{k-1} \beta_{k} l_{k} \beta_{k+1} l_{k+1}\right\rangle_{l_{k-1}}^{l_{k}} S_{l_{k+1}}^{l_{k}^{\prime}}\left(\beta_{k}-\beta_{k+1}\right)=\left|\cdots l_{k-1} \beta_{k+1} l_{k}^{\prime} \beta_{k} l_{k+1} \cdots\right\rangle .
$$

These amplitudes can be expressed in terms of $q$-analogos of $6-j$ symbols [11] and coincide with critical RSOS heights [10-12]. Without going into details we present explicit formulas for the amplitudes:

$$
\begin{aligned}
& { }_{l}^{l+(1 / 2)} S_{l+1}^{l+(1 / 2)}(\beta)={ }_{l}^{l-(1 / 2)} S_{l-1}^{l-(1 / 2)}(\beta) S_{0}(\beta), \\
& { }_{l}^{l+(1 / 2)} S_{l}^{l+(1 / 2)}(\beta)=\frac{\operatorname{sh} \frac{\pi}{\xi}(\beta+(2 l+1) \pi i) \sin \frac{\pi^{2}}{\xi}}{\sin \frac{\pi^{2}}{\xi}(2 l+1) \operatorname{sh} \frac{\pi}{\xi}(\beta-\pi i)} S_{0}(\beta), \\
& l_{l}^{l-(1 / 2)} S_{l}^{l-(1 / 2)}(\beta)=\frac{\operatorname{sh} \frac{\pi}{\xi}(\beta-(2 l+1) \pi i) \sin \frac{\pi^{2}}{\xi}}{\sin \frac{\pi^{2}}{\xi}(2 l+1) \operatorname{sh} \frac{\pi}{\xi}(\beta-\pi i)} S_{0}(\beta) \\
& l_{l}^{l+(1 / 2)} S_{l}^{l-(1 / 2)}(\beta)=l_{l}^{l-(1 / 2)} S_{l}^{l+(1 / 2)}(\beta)=\frac{\operatorname{sh} \frac{\pi}{\xi} \beta\left(\sin \left(\frac{\pi^{2}}{\xi} 2 l\right) \sin \left(\frac{\pi^{2}}{\xi}(2 l+2)\right)\right)^{1 / 2}}{\sin \frac{\pi^{2}}{\xi}(2 l+1) \operatorname{sh} \frac{\pi}{\xi}(\beta-\pi i)} S_{0}(\beta),
\end{aligned}
$$


the square root in the last line is understood as

$$
\begin{aligned}
\left(\sin \frac{\pi^{2} 2 l}{\xi} \sin \frac{\pi^{2}(2 l+2)}{\xi}\right)^{1 / 2}= & \sqrt{\operatorname{sgn}\left(\sin \frac{\pi^{2} 2 l}{\xi} \sin \frac{\pi^{2}(2 l+2)}{\xi}\right)} \\
& \cdot\left(\left|\sin \frac{\pi^{2} 2 l}{\xi} \sin \frac{\pi^{2}(2 l+2)}{\xi}\right|\right)^{1 / 2}, \quad \sqrt{-1}=i .
\end{aligned}
$$

Other matrix elements of $S$-matrix are equal to zero. From the definition of the $S$-matrix as restricted SG one it follows that

$$
\sum_{k_{2}}^{l_{2}} l_{1}^{k_{l_{3}}} S_{l_{2}}(\beta)_{l_{1}}^{k_{2}} S_{l_{3}}^{l_{2}^{\prime}}(-\beta)=\delta_{l_{2} l_{2}^{\prime}}
$$

i.e., the matrix $S(-\beta)$ is inverse to $S(\beta)$. The Eq. (2.6) can be also checked by straight calculations using (2.5).

The RSOS structure of $S$-matrix in the restricted SG model was discussed also in [24].

Our goal is to check the unitary of the $S$-matrix. To this end we have obviously to compare $S^{\star}(\beta)$ and $S(-\beta)$. Evidently, everything depends on the square roots in (2.5). Simple reasonings show that in general we have the following property of $S$-matrix:

$$
A S^{\star} A S=S A S^{\star} A=I,
$$

where $A$ is the operator defined by the formula

$$
\begin{aligned}
A\left|\beta_{n} l_{n-1} \cdots \beta_{1}\right\rangle & =\varepsilon\left(l_{1} \cdots l_{n-1}\right)\left|\beta_{n} l_{n-1} \cdots \beta_{1}\right\rangle, \\
\varepsilon\left(l_{1} \cdots l_{n-1}\right) & =\operatorname{sgn}\left(\prod_{j=1}^{n-1} \sin \frac{l_{j} \pi^{2}}{\xi}\right) .
\end{aligned}
$$

The symbol $\varepsilon\left(l_{1} \cdots l_{n-1}\right)$ coincides with that introduced in Sect. 0 .

Certain reasonings based on the exact formulas for the formfactors show also that

$$
\mathscr{T}_{\mu \nu}^{\star}=A \mathscr{T}_{\mu \nu} A, \quad \psi^{\star}=A \psi A
$$

where $\psi=\exp (i \sqrt{\gamma} \varphi / 2)$.

Let us discuss these results. First, let $\xi>\pi$. In this case $A=I$ only if $\xi=\pi$ which means that $q=\exp (2 \pi i / r)$. For these values of $\xi$ we obtain self-consistent physical theories with unitary $S$-matrices and selfadjoint operators $J_{\mu \nu}, \psi$. We would like to notice here the two simplest cases: $\xi=2 \pi, \xi=3 \pi$. For $\xi=2 \pi$ the limitation $l_{i} \leqq 0$ means that $H_{2}=\varnothing$. For SG theory (2.3) it means that for $\xi=2 \pi$ all the Green functions of the $T_{R}$-invariant operators are equal to zero. For $\xi=3 \pi$ the internal degrees of freedom appear to be frozen: $l_{i} \leqq \frac{1}{2}$, hence for $l_{1} \cdots l_{n-1}$ we have only one possibility $l_{1} l_{2} l_{2} \cdots=\frac{1}{2}, 0, \frac{1}{2} \cdots$. That is why the model RSG(3) can be interpreted as a theory with one scalar particle. Correspondingly the $S$-matrix is equal to -1 . So, $\operatorname{RSG}(3)$ can be identified with scaling Ising model, the operator $\psi$ corresponding to the disorder parameter. Going back to SG we realize that for $\xi=3 \pi$ the Green functions of $T_{R}$-invariant operators coincide with those in the scaling Ising model. 
There is one more case with unitary $S$-matrix for $\xi>\pi: \xi=(3 \pi / 2), q=\exp (4 \pi i / 3)$. In this case the internal degrees of freedom are frozen: $l_{i} \leqq \frac{1}{2}$. The operator $A$ acts as follows:

$$
A\left|\beta_{2 n} \frac{1}{2} \beta_{2 n-1} 0 \cdots \beta_{1}\right\rangle=(-1)^{n}\left|\beta_{2 n} \frac{1}{2} \beta_{2 n-1} 0 \cdots \beta_{1}\right\rangle .
$$

This operator cannot manifest itself on-shell because of the pure elastic character of scattering, on the $S$-matrix is unitary:

$$
S S^{\star}=I .
$$

But the operator $A$ is essential off-shell where the states with a different number of particles are mixed: the operators $\mathscr{T}_{\mu \nu}$ and $\psi$ are not selfadjoining and cannot be made selfadjoint since they are not mutually local with their adjoints. In this case we have to algebras of local operators $\mathscr{A}$ and $\mathscr{A}^{*}$ which are mutually local. This situation was discussed in [9]; we call it $*$-violated. The internal degrees of freedom in the model are frozen; that is why the spectrum can be interpreted as containing one scalar particle. Corresponding $S$-matrix is

$$
S(\beta)=\frac{1}{i} \operatorname{th} \frac{1}{2}\left(\beta-\frac{\pi i}{2}\right) \text {. }
$$

This is the simplest factorizable $S$-matrix. The sign "_" occurring in the crossing relation for this $S$-matrix reflects the $*$-violation of the theory.

Consider now the range $\xi<\pi$. Here breathers appear. One can show that in that case the operator $A$ in (2.8) should be replaced by $A C$, where $C$ is the breathers $C$-conjugation operator which gives $1(-1)$ on even (odd) breathers states. For $\xi<\pi$ unitary $S$-matrices appear when $\xi=\pi /(k+1), q=\exp (2 \pi i / 2)$, and when $\xi=3 \pi / 3 k+2, q=\exp (4 \pi i / 3)$, the solitons states are frozen and in solitons subspaces $A$ is the same as in (2.9). All these models are $*$-violated because $A$ is changed to $C A$, and the operators $\mathscr{T}_{\mu \nu}, \psi$ necessary mix odd and even breathers states. Certainly the soliton-breather and breather-breather $S$-matrices being scalar ones coincide with those in SG theory.

It is interesting to list the models with scalar $S$-matrices. For $\xi>\pi$ there are two theories of the kind: $\xi=3 \pi, \xi=3 \pi / 2$ considered above. For $\xi<\pi$ there are three series. The first one: $\xi=2 \pi /(2 n+1), q=-1$ [9] solitons disappear completely, only breathers remain, and their $S$-matrices are known. The second one: $\xi=3 \pi /(3 n+1), q=\exp (2 \pi i / 3)$, solitons are frozen and give one particle. The $S$-matrix of these particles is

$$
S(\beta)=(-1)^{n+[(n+1) / 2]} \prod_{q=1}^{n} \frac{\operatorname{th}\left(\frac{1}{2}\left(\beta-\frac{3 i \pi q}{3 n+1}\right)\right)}{\operatorname{th}\left(\frac{1}{2}\left(\beta+\frac{3 i \pi q}{3 n+1}\right)\right)} \prod_{m=1}^{n} \operatorname{th}\left(\frac{1}{2}\left(\beta-\frac{i \pi}{3 n+1}(3 n+2-3 m)\right)\right) .
$$

Scattering of this particle on breathers is described by the usual soliton-breather $S$-matrix, scattering of breathers is described by the usual breathers $S$-matrix. The third one: $\xi=3 \pi /(3 n+2), q=\exp (4 \pi i / 3)$, everything is similar to the previous case, 
the $S$-matrix of soliton-made particle is

$$
S(\beta)=(-1)^{n+1+[n / 2]} \prod_{q=1}^{n} \frac{\operatorname{th}\left(\frac{1}{2}\left(\beta-\frac{3 i \pi q}{3 n+2}\right)\right)}{\operatorname{th}\left(\frac{1}{2}\left(\beta+\frac{3 i \pi q}{3 n+2}\right)\right)} \prod_{m=0}^{n} \operatorname{th}\left(\frac{1}{2}\left(\beta-\frac{i \pi}{3 n+2}(3 n+1-3 m)\right)\right)
$$

Let us summarize the results of this section. If we consider the Green functions of invariant operators for rational values of $(\xi / \pi)$ the space of states automatically appears to be reduced. For the reduced model self-consistent physical interpretation is possible for

$$
\xi=\frac{r \pi}{r k+1} r=2, \ldots, k=0,1, \ldots, \xi=\frac{3 \pi}{3 k+2} k=0,1, \ldots
$$

only in these cases the $S$-matrices are unitary. The models $\mathrm{RSG}(r)$ are the only models possessing the selfadjoint algebra of local operators. Other models

$$
\left(\operatorname{RSG}\left(\frac{r \pi}{k r+1}\right) \text { for } k \geqq 1, \quad \operatorname{RSG}\left(\frac{3 \pi}{3 k+2}\right)\right)
$$

are $*$-violated which means that their algebras of local operators are not closed with respect to the Hermitian conjugation.

\section{Ultraviolet limit of RSG}

Let us discuss the ultraviolet behavior of the model RSG(2/2 $)$. Unfortunately we cannot yet extract the precise information about the behavior of the Green functions at short distances from the formfactors expansion. In the paper [9] it is shown that the formfactors possess remarkable properties which allow to write down series for scaling dimensions which is far more effective than the direct investigation of short distance behavior of Green functions. However, the exact summation of these series remains an open problem. Thus in discussing ultraviolet behavior we have to rely on physical speculations.

The $T_{R}$ invariant energy-momentum tensor is modified energy-momentum tensor

$$
\tilde{T}_{\mu \nu}=T_{\mu \nu}+i 2^{-5 / 2} \frac{\sqrt{\gamma}}{\xi} \varepsilon_{\mu \mu^{\prime}} \varepsilon_{v v^{\prime}} \partial_{\mu^{\prime}} \partial_{\nu^{\prime}} \varphi .
$$

Corresponding central charge is

$$
c=1-6\left(\frac{\gamma}{8 \pi}+\frac{8 \pi}{\gamma}-2\right)
$$

because the central charge of $T_{\mu \nu}$ is equal to 1 as well as the coefficient before the $\delta$-function in the commutator $\left[\varphi, \partial_{0} \varphi\right][15]$. The formula holds in reduced theory if $\xi / \pi$ is rational because the Green functions of reduced theory coincide with those 
in SG. So, it is natural to suppose that the reduction of SG to $\operatorname{RSG}\left(r / r_{1}\right)$ is an analog of Dotsenko-Fateev construction [21] for massless fields. In more adequate terms in the context of the present paper, this construction is described in [22] (as reduction of the space of states of the massless free boson field which leads to the minimal models of CFT). Having in mind this analogy and the formula for the central charge (3.1) we suppose that the ultraviolet limit of $\operatorname{RSG}\left(r / r_{1}\right)$ is the minimal CFT $M_{p_{1} / p_{2}}\left(p_{1}=r, p_{2}=r_{1}+r\right)$ [1]. So we adopt the following scheme:

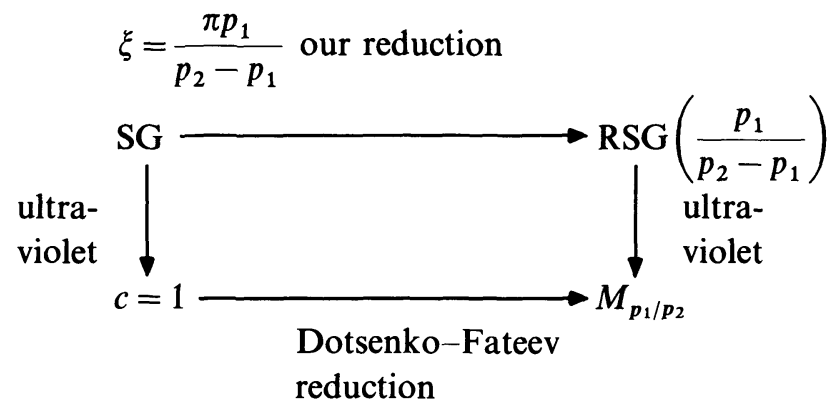

Then the scaling dimensions of the operators $J_{\mu \mu} \sim P_{r}^{\prime} \exp (i \sqrt{\gamma} \varphi) P_{r}, \psi=$ $P_{r}^{\prime} \exp (i \sqrt{\gamma} \varphi / 2) P_{r}$ should be equal to

$$
\begin{gathered}
\frac{p_{1}}{p_{2}}-\sqrt{\frac{p_{1}}{p_{2}} \frac{\left(p_{2}-p_{1}\right)^{2}}{p_{1} p_{2}}}=\frac{2 p_{1}-p_{2}}{p_{2}}, \\
\frac{p_{1}}{4 p_{2}}-\sqrt{\frac{p_{1}}{4 p_{2}} \frac{\left(p_{2}-p_{1}\right)^{2}}{p_{1} p_{2}}}=\frac{3 p_{1}-2 p_{2}}{4 p_{2}},
\end{gathered}
$$

i.e., in scaling limit these operators coincide with the operators $\phi_{1,3}, \phi_{1,2}$, respectively. It means that from the viewpoints of papers [5-7] $\operatorname{RSG}\left(p_{1} / p_{2}-p_{1}\right)$ is the perturbation of $M_{p_{1} / p_{2}}$ via the operator $\phi_{1,3}$. One can add other arguments in favor of this conclusion: i) RSG (3) coincides with the scaling Ising model, ii) it is possible to calculate scaling characteristics of the models studying their low-temperature thermodynamics in the spirit of papers [23,25]. However, mathematically complete reasonings involving the direct investigation of short distance singularities of Green functions is missed yet.

The conclusion that $\operatorname{RSG}\left(p_{1} / p_{2}-p_{1}\right)$ is the perturbation of $M_{p_{1} / p_{2}}$ by the operator $\phi_{1,3}$ implies the following nice picture: for the SG theory two scaling limits are possible which lead to the theories $c=1$ and $c=1-6\left(p_{2}-p_{1}\right)^{2} / p_{1} p_{2}$. Let us explain. Consider, for example, the operator $\mathscr{T}_{\mu \mu}$. This operator has scaling dimension $\Delta_{13}=\left(2 p_{1}-p_{2}\right) / p_{2}$, i.e.,

$$
\left\langle 0\left|\mathscr{T}_{\mu \mu}(z, \bar{z}) \mathscr{T}_{\mu \mu}(0,0)\right| 0\right\rangle \sim_{\rho=(z \bar{z})^{1 / 2} \rightarrow 0} \text { const } M^{4} \frac{1}{(M \rho)^{4 \Delta_{13}}}+\cdots,
$$

$M$ is the mass of soliton. 
The formulas (2.3), (1.9) mean that

$$
\left\langle 0\left|\mathscr{T}_{\mu \mu}(z, \bar{z}) \mathscr{T}_{\mu \mu}(0,0)\right|\right\rangle=\text { const } M^{4}\langle 0| \exp (i \sqrt{\gamma} \varphi(z \bar{z})) \exp (i \sqrt{\gamma} \varphi(0,0)|0\rangle .
$$

where the left-hand side is RSG Green function, the right-hand side is SG Green function. Hence,

$$
\langle 0| \exp \left(i \sqrt{\gamma} \varphi(z \bar{z}) \exp (i \sqrt{\gamma} \varphi(0,0))|0\rangle \sim_{\rho \rightarrow 0} \frac{1}{(M \rho)^{4 \Delta_{13}}}+\cdots .\right.
$$

At the same time one has the formula

$$
\langle 0| \exp \left(i \sqrt{\gamma} \varphi(z \bar{z}) \exp (-i \sqrt{\gamma} \varphi(0,0))|0\rangle \sim_{\rho \rightarrow 0} \frac{1}{(M \rho)^{4 \Delta}},\right.
$$

where $\Delta=p_{1} / p_{2}$. The last formula from the fact that in ultraviolet limit SG leads to $c=1$ theory.

To get the scaling limit leading to $c=1$ theory one has first to rescale the operators $\exp (i \sqrt{\gamma} \varphi), \exp (-\sqrt{\gamma} \varphi)$,

$$
v=m^{2 \Delta} \exp (i \sqrt{\gamma} \varphi), \quad v^{*}=m^{2 \Delta} \exp (-i \sqrt{\gamma} \varphi),
$$

where $m$ has the dimension of mass. Then one considers the limit $\rho \rightarrow 0, m \rightarrow 0$, $r=(M / m) \rho$ is finite in all the Green functions. In particular in this limit one gets from (3.2), (3.3):

$$
\begin{gathered}
\left\langle 0\left|v(z, \bar{z}) v^{*}(0,0)\right| 0\right\rangle=\frac{1}{r^{4 \Delta}}, \\
\langle 0|v(\xi \bar{\xi}) v(0,0)| 0\rangle=0, \quad \xi=\frac{z M}{m}
\end{gathered}
$$

because $\Delta_{13}<\Delta$. These Green functions correspond to $c=1$ theory. A part of the information about the behavior of the Green functions at short distances is massive theory (for example the formula (3.2)) is missed in this scaling limit.

The $S L(2)_{q}$ symmetry of SG allowss to extract from the operator algebra the subalgebra of invariant operators which contain $\exp (i \sqrt{\gamma} \varphi)$ but does not contain $\exp (-i \sqrt{\gamma} \varphi)$. For this subalgebra one can consider another scaling limit.

$$
v=m^{2 \Delta_{13}} \exp (i \sqrt{\gamma} \varphi)
$$

$m \rightarrow 0, \rho \rightarrow 0, r=M \rho / m$ is finite. In that limit we get

$$
\langle 0|v(\xi, \bar{\xi}) v(0,0)| 0\rangle=\frac{1}{r^{4 \Delta_{13}}}, \quad \xi=\frac{M z}{m} .
$$

The resulting algebra of operators should coincide with that of the $M_{p_{1} / p_{2}}$ model.

Thus the short distance behavior of different SG Green functions is described by diferent $C F T$. Certainly there are Green functions for which both scaling limits give no information, for example

$$
\langle 0| \exp i \sqrt{\gamma} \varphi\left(z_{1} \bar{z}_{1}\right) \exp \left(i \sqrt { \gamma } \varphi ( z _ { 2 } \overline { z } _ { 2 } ) \operatorname { e x p } \left(-i \sqrt{\gamma} \varphi\left(x_{3} \bar{z}_{3}\right)|0\rangle .\right.\right.
$$


For this Green function the first scaling limit gives zero, the second scaling limit is not applicable because $\exp (-i \sqrt{\gamma} \varphi)$ is not $T_{R^{-}}$-invariant.

\section{Conclusion}

We considered $S L(2)_{q}$ symmetry in the SG model. More precisely we used a regularization in the region $4 \pi \leqq \gamma \leqq 8 \pi$ such that the model becomes equivalent to a massless two-component Thirring model with anisotropical interaction [8]. For these values of the coupling constant there is no unique ultraviolet regularization. An example of a different regularization is given in [27]. It would be interesting to understand the hidden quantum group symmetry in this case.

The Sine-Gordon model is a particular case of the so-called two-dimensional Toda models. Each of these models is characterized by a massive parameter, $m$, a massless parameter $\gamma$ and by some simple Lie algebra G. This model describes $n=$ rank of interacting scalar fields $\varphi^{a}, a=1, \ldots, n$ with Lagrangian [28],

$$
\mathscr{L}=\int\left(\sum_{a=1}^{n}\left(\partial_{\mu} \varphi^{a}\right)^{2}+\frac{m^{2}}{\gamma} \sum_{i=1}^{n+1} q_{i}\left[\exp \left(\sum_{a=1}^{n} \sqrt{-\gamma} \alpha_{i}^{a} \varphi^{2}\right)-1\right]\right) d x
$$

where $\left\{\alpha_{i}\right\}_{i=1}^{n}$ are the simple root of $\mathbf{G}, \alpha_{n+1}$ is the maximal negative root, $\alpha_{n+1}=-\sum_{i=1}^{n} q_{i} \alpha_{i}, q_{i}>0$.

For negative $\gamma$ these models have hermitian Hamiltonian, massive excitations and well-defined scattering theory. For positive $\gamma$-s the Hamiltonian is not a hermitian operator (except the case $\mathbf{G}=s l_{2}$ corresponding to the SG model). But precisely this case seems to be most interesting from our point of view. In this case it can be shown that the Hamiltonian of the model is invariant under the hidden $G_{q}$ (and $G_{\bar{q}}$ ). For rational $\gamma / \pi$ there is a restriction on the operator algebra similar to the one described above for the $S G$ model. This restriction becomes more interesting for $\gamma=(8 \pi p) / p+1$ (at least for $\mathbf{G}=A_{n}, D_{n}, E_{6-8}$. In this case one can show that on the restricted operator algebra there is a new *-involution. The restricted theories are unitary under this involution and we expect that they will be massive integrable perturbations of conformal field theories with the following central charges [29]:

$$
\begin{array}{ll}
A_{n-1}^{(p)}, c_{p}=(n-1)\left(1-\frac{n(n-1)}{p(p+1)}\right), & p=n+1, n+2, \ldots, \\
\mathscr{D}_{n}^{(p)}, c_{p}=n\left(1-\frac{(2 n-2)(2 n-1)}{p(p+1)}\right), & p=2 n-1,2 n, \ldots, \\
E_{6}^{(p)}: c_{p}=6\left(1-\frac{12 \cdot 13}{p(p+1)}\right), & p=13,14, \ldots, \\
E_{7}^{(p)}: c_{p}=7\left(1-\frac{18 \cdot 19}{p(p+1)}\right), & p=18,19, \ldots, \\
E_{8}^{(p)}: c_{p}=8\left(1-\frac{30 \cdot 31}{p(p+1)}\right), & p=31,32, \ldots
\end{array}
$$

We hope to describe these models in more detail in a separate publication. 
Acknowledgements. We would like to thank A. Kirillov and A. Zamolodchikov for stimulating discussions.

\section{References}

1. Belavin, A. A., Polyakov, A. M., Zamolodchikov, A. B.: Nucl. Phys. B241, 333 (1984)

2. Takhtajan, L. A., Faddeev, L. D.: Teor. Mat. Fiz. 21, 160 (1974)

3. Sklyanin, E. K., Takhtajan, L. A., Faddeev, L. D.: Teor. Mat. Fiz. 40, 194 (1979)

4. Zamolodchikov, A. B., Zamolodchikov, Al. B.: Ann. Phys. 120, 253 (1979)

5. Zamolodchikov, A. B.: Pisma ZhETF 46, 129 (1987)

6. Zamolodchikov, A. B.: Int. J. Mod. Phys. A.

7. Zamolodchikov, A. B.: Talk given at Tanigushi Conference, Kysto, 1988, to be published

8. Luther, A., Emery, V. J.: Phys. Rev. Lett. 33, 589 (1974)

9. Smirnov, F. A.: Reductions of Quantum Sine-Gordon Model as Perturbations of Minimal Models of Conformal Field'Theory, Preprint LOMI E-4-89 (1989)

10. Andrews, G. E., Baxter, R. J., Forrester, P. J.: J. Sat. Phys. 35, 193 (1984)

11. Date, E, Jimbo, M., Miwa, T., Ocado, M.: Lett Math. Phys. 12, 209 (1986)

12. Bazhanov, V., Reshetikhin, N.: Int. J. Mod. Phys. A4, 115 (1989)

13. Friedan, D., Qui, Z., Shenker, S.: Phys. Rev. Lett. 52, 1575 (1984)

14. Drinfeld, V. G.: Proceedings of the International Congress of Mathematicians, vol. 1, pp. 798-820. Berkeley, California, New York: Academic Press 1986

15. Woronowicz, S.: Publ. RIMS, Kyoto University, 23, 117-181 (1987)

16. Fadeev, L., Reshetikhin, N., Takhtajan, L.: Quantization of Lie Groups and Lie Algebras, LOMI-preprint, E-14-87 (1987); extended version: Algebra and Analysis, 1, N1 (1989), Leningrad (in Russian)

17. Kirillov, A. N., Reshetikhin, N. Yu.: Representations of the Algebra $U_{9}(s(2))$, q-orthogonal Polynomials and Invariants of Links, Preprint LOMI E-9-88 (1988)

18. Kirillov, A. N., Smirnov, F. A.: Phys. Lett. B198, 506 (1987)

19. Smirnov, F. A.: Formfactors in Completely Integrable Models of QFT, to be published in World Scientific

20. Smirnov, F. A.: J. Phys. 17A, L873 (1984)

21. Dotsenko, H. S., Fateev, V. A.: Nucl. Phys. B240, 312 (1984)

22. Felder, G.: Nucl. Phys. B317, 215 (1989)

23. Blöte, H. W., Cardy, J., Nightingale, M. P.: Phys. Rev. Lett. 56, 742 (1986)

24. LeClair, A.: Restricted Sine-Gordon Theory and the Minimal Conformal Seires, PUPT-1124; Bazhanov, V., and Reshetikhin, N.: unpublished

25. Cardy, J.: Nucl. Phys. B270, 186 (1986)

26. Turaev, V., Reshetikhin, N.: Invariants of 3-manifolds via Link Polynomials and Quantum Groups. Commun. Math. Phys. 127, 1 (1990)

27. Izergin, A. G., Korepin, V. E.: Nucl. Phys. B205, 401 (1982)

28. Mikhailov, A., Olshanetsky, M., Perelomov, A.: Commun. Math. Phys. 79, 473 (1981)

29. Lukyanov, S., Fateev, V.: Lectures given in II. Kiev Spring School "Contemporary Problems in Theoretical Physics," Kiev 1988, Preprint ITF-88-74-76P, Kiev 1988 (in Russian)

Communicated by A. Jaffe

Received November 30, 1989 
